\title{
Targeting neurosteroidogenesis as therapy for PTSD
}

\section{Graziano Pinna *}

Psychiatric Institute, Department of Psychiatry, University of Illinois at Chicago, Chicago, IL, USA

*Correspondence: gpinna@psych.uic.edu

Edited by:

Thibault Renoir, Florey Institute of Neuroscience and Mental Health, Australia

Reviewed by:

Jamie Maguire, Tufts University School of Medicine, USA

Rainer Rupprecht, University Regensburg, Germany

Keywords: neurosteroidogenesis, PTSD, PTSD treatment, GABAergic neurotransmission, anti-PTSD drug discovery, selective brain steroidogenic stimulants (SBSSs), allopregnanolone, ganaxolone

Posttraumatic stress disorder (PTSD) is a severe condition resulting from exposure to traumatic events, such as combat situations, sexual assault, serious injury or the threat of death. Symptoms include disturbing recurring flashbacks, avoidance or numbing of memories of the event, and hyperarousal, which continue for more than a month after the traumatic event. Reduced cortical GABA (Kugaya et al., 2003) and cebrospinal fluid (CSF) allopregnanolone levels (Rasmusson et al., 2006) that positively and allosterically modulate GABA action at $\mathrm{GABA}_{\mathrm{A}}$ receptors (Belelli and Lambert, 2005) suggest that in PTSD patients, a perturbation of GABAergic neurotransmission plays a role in the pathogenesis of this disorder. Thus restoring downregulated brain allopregnanolone levels may be beneficial in treating PTSD.

There is a general consensus that maladaptive fear responses (i.e., impaired fear extinction) are a core feature of stressinduced PTSD (Myers and Davis, 2007; Maren, 2008). Exaggerated fear responses and impaired extinction learning, or the inability to extinguish fear memories, are often treated with exposure-based therapy (EBT), which involves the exposure of the patient to the feared context without any danger (Joseph and Gray, 2008). This closely approximates the procedure used to simulate and study fear responses and fear extinction learning in PTSD mouse models (Marks, 1979). While psychological therapy has been highly effective both in treating PTSD and in preventing the progression of the event sequelae that leads to consolidation of fear memories, one challenge of PTSD therapy is the spontaneous recovery of fear that often reemerges following successful EBT.

For this reason, pharmacological treatment may be advantageous alone or in combination with EBT. Selective serotonin reuptake inhibitors (SSRIs) are currently the drugs of choice in treating PTSD. They are effective in facilitating and restoring the neurobiological changes altered in PTSD patients, and they are devoid of the unwanted side effects that plague the use of benzodiazepines, more importantly, SSRIs are potent therapeutics where benzodiazepines fail to be beneficial. Following the observation that low non-serotonergic doses of fluoxetine and congeners increase allopregnanolone levels as their primary mechanism of action, we suggested that SSRIs acting as selective brain steroidogenic stimulants (SBSSs) can improve dysfunctional emotional behavior and may be of advantage in PTSD treatment. In addition to its use in PTSD, this novel steroidogenic mechanism of action of SSRIs given at low doses offers enormous therapeutic potentials for the treatment of other psychiatric disorders, including anxiety spectrum disorders, premenstrual dysphoria, and probably depression, as these disorders may be caused by a downregulation of neurosteroid biosynthesis (Uzunov et al., 1996; Westenberg, 1996; Guidotti and Costa, 1998; Romeo et al., 1998; Uzunova et al., 1998; Steiner and Pearlstein, 2000; Berton and Nestler, 2006; Pinna et al., 2006a, 2009; Pinna, 2010; Ipser and Stein, 2012; Pinna and Rasmusson, 2012; Lovick, 2013).

In vitro studies show that SSRIs may activate $3 \alpha$-hydroxysteroid dehydrogenase, thereby facilitating the reduction of $5 \alpha$-dihydroprogesterone into allopregnanolone (Griffin and Mellon, 1999). Nonetheless, the precise neuronal mechanisms involved in the neurosteroidogenic action of SSRIs remain unclear. Drug design welcomed allopregnanolone biosynthesis as a target for novel rapidly acting anxiolytics devoid of sedation, tolerance, and withdrawal liabilities (Rupprecht et al., 2009, 2010; Schüle et al., 2011), and, in addition to low doses of SSRIs, selective ligands for the $(18 \mathrm{kDa})$ translocase protein (TSPO), which increase allopregnanolone levels, may be beneficial in anxiety and PTSD (Rupprecht et al., 2009).

\section{A PTSD MOUSE MODEL}

In our laboratory, we have used the socially isolated (SI) mouse as a model characterized by a downregulation of allopregnanolone biosynthesis associated with endophenotypic features of PTSD. The relevance of the SI mice as a model of PTSD lays in reproducing behavioral and neurochemical alterations that are found in PTSD patients (Pibiri et al., 2008). Thus, SI mice express decreased corticolimbic allopregnanolone levels in emotion-relevant brain areas (frontal cortex, hippocampus, basolateral amygdala) (Pibiri et al., 2008; Pinna et al., 2008). The impulsivity and violence of combat veterans (Forbes et al., 2008), is matched in SI mice by high levels of aggression (Pinna et al., 2003). In PTSD patients, enhanced contextual fear and impaired fear extinction learning was shown during re-exposure to events that symbolize the triggering traumatic event; however, cued fear was not changed (Ameli et al., 2001; Rauch et al., 2006). SI mice, analogously, display exaggerated contextual fear and impaired 
fear extinction and unchanged cued fear responses (Pibiri et al., 2008; Pinna et al., 2008). Interestingly, PTSD patients fail to respond to the pharmacological action of benzodiazepine and show decreased frontocortical benzodiazepine site binding (Bremner et al., 2000). Of note, SI mice show a lack of sedative/anxiolytic activity to diazepam and zolpidem (Pinna et al., 2006b; Nin et al., 2011). In contrast, allopregnanolone or S-norfluoxetine at low, non-SSRI active doses reduced anxiety in SI mice, an effect that was mimicked by allopregnanolone's analog ganaxolone (Pinna and Rasmusson, submitted). Interestingly, anxiolytic doses of S-norfluoxetine also normalized the immobility time of SI mice as determined by the forced swim test (Nin et al., 2011).

Social isolation causes changes in the frontocortical and hippocampus expression of $\mathrm{GABA}_{\mathrm{A}}$ receptor subunits. The cortical expression of $\alpha 1, \alpha 2$, and $\gamma 2$ subunit mRNA was decreased by $\approx 50 \%$, and $\alpha 4$ and $\alpha 5$ was increased by $130 \%$ in SI mice. The expression $\alpha 1$ subunit mRNA in layer I was decreased by $50 \%$ and unchanged in layer V of SI mice (Pinna et al., 2006b). Likewise, $\mathrm{GABA}_{\mathrm{A}}$ receptor subunit expression of $\alpha 1$ was decreased and that of $\alpha 5$ was increased in the hippocampus. A downregulation of $\alpha 1(-40 \%)$ and an increase in the expression of $\alpha 5$ subunit proteins $(+100 \%)$ was also determined in SI mice. Because $\gamma 2$ subunits are a necessary prerequisite for the formation of benzodiazepine-sensitive $\mathrm{GABA}_{\mathrm{A}}$ receptors, our study suggests that the decrease in $\gamma 2$ expression and the lack of benzodiazepine's anxiolytic action observed in SI mice may be a result of stressinduced formation of benzodiazepineinsensitive $G_{A B A}$ receptors strategically integrated in circuitry that regulate anxiety. Interestingly, we observed a decreased benzodiazepine binding to hippocampal synaptic membranes (Pinna et al., 2006b).

Unlike benzodiazepines, which have a selective pharmacological profile and fail to activate $\mathrm{GABA}_{\mathrm{A}}$ receptors containing $\alpha 4$ and $\alpha 6$ subunits (Brown et al., 2002), allopregnanolone modulation of $\mathrm{GABA}_{\mathrm{A}}$ receptors exhibits a broad pharmacological profile. Although allopregnanolone acts preferentially on $\delta$ subunit-containing $\mathrm{GABA}_{\mathrm{A}}$ receptors, which confers neurosteroid sensitivity, it also exerts effects on other $\mathrm{GABA}_{\mathrm{A}}$ receptor subtypes at higher concentrations (Mihalek et al., 1999; Stell et al., 2003). Thus, increasing corticolimbic allopregnanolone levels with allopregnanolone injections or stimulating allopregnanolone biosynthesis with S-norfluoxetine, or directly activation of $\mathrm{GABA}_{\mathrm{A}}$ receptors with ganaxolone likely improved anxiety because allopregnanolone/ganaxolone acts on a larger spectrum of $\mathrm{GABA}_{\mathrm{A}}$ receptor subunits. Thus, allopregnanolone or analogs are more advantageous than benzodiazepines because they improve anxiety, fear, and aggressiveness when benzodiazepines are inactive. In addition, unlike benzodiazepines, allopregnanolone, ganaxolone, or SBSS ligands may improve emotional behavior at non-sedative concentrations (Pinna et al., 2003, 2006b; Nelson and Pinna, 2011; Nin et al., 2011; Pinna and Rasmusson, submitted). These observations suggest that drugs designed to selectively increase neurosteroidogenesis may alleviate PTSD by facilitating $\mathrm{GABA}_{\mathrm{A}}$ receptor neurotransmission.

\section{PHARMACOLOGICAL TARGETS TO STIMULATE NEUROSTEROIDOGENESIS}

A seminal observation by Uzunova et al. (1998) suggested that SSRIs, including fluoxetine and fluvoxamine might be beneficial in the treatment of major unipolar depression by increasing the brain levels of allopregnanolone. This SSRI-induced neurosteroidogenic effect correlated with improved depressive symptomatology and was confirmed by several other reports in the field (Romeo et al., 1998, reviewed in Pinna et al., 2006a; Schüle et al., 2011). Previous studies reported that SSRIs induce allopregnanolone biosynthesis in rodent brain slices following incubation with the allopregnanolone's precursor $5 \alpha$ dihydroprogesterone (Uzunov et al., 1996). These observations were confirmed in experiments in which fluoxetine's ability to induce neurosteroidogenesis in several corticolimbic structures was challenged using mouse models of psychiatric disorders such as the SI mouse (Pinna et al., 2003, 2004). Interestingly, fluoxetine's action as a steroidogenic stimulant appeared to be the primary mechanism of SSRIs: the drug concentrations, which increased brain allopregnanolone levels were less than, and dissociated from, those effective as a selective serotonin reuptake inhibitor, which justified a new name to better define the "SSRI" mechanism of action: selective brain steroidogenic stimulants or SBSS (Pinna et al., 2006a, 2009). The discovery of this novel mechanism of action of SSRIs has stimulated drug design to focus on the development of new, more effective therapies for anxiety disorders by targeting neurosteroidogenesis. Novel neuronal biomarkers, for the pharmacological target of neurosteroidogenesis as the next generation of anxiolytic drugs, have been discovered (Rupprecht et al., 2009). These include the TSPO (Costa et al., 1994; Papadopoulos et al., 2006), which represents the starting point and an important rate-limiting step in neurosteroidogenesis. TSPO regulates neurosteroidogenesis in the brain by gating the entry of cholesterol into the inner mitochondrial membranes of glial cells, and its conversion into pregnenolone by P450scc Figure 1 (Costa and Guidotti, 1991; Costa et al., 1994; Papadopoulos et al., 2006; Rupprecht et al., 2010). Pregnenolone can then be taken up by pyramidal neurons (Costa and Guidotti, 1991) where a cascade of enzymatic processes takes place in the cytosol resulting in the production of neurosteroids, including pregnenolone sulfate and allopregnanolone Figure 1. New molecules that bind with high affinity to TSPO have been recently investigated; these drugs are able to exert important anxiolytic effects but are devoid of the unwanted side effects associated with benzodiazepines, including over-sedation, tolerance, and withdrawal symptoms (Rupprecht et al., 2009, 2010). In mouse models, TSPO agents have been shown to potently increase allopregnanolone levels in the hippocampus and cortex, as well as to induce anxiolytic effects (Kita et al., 2004). XBD173 and etifoxine have proven to be highly efficacious anxiolytic and antidepressant drugs in a number of behavioral tests (Rupprecht et al., 2010; Schüle et al., 2011). The anxiolytic effects of these agents were related to their ability to increase neurosteroid biosynthesis upstream of allopregnanolone synthesis within the neurosteroidogenic cascade Figure 1, as confirmed by studies 


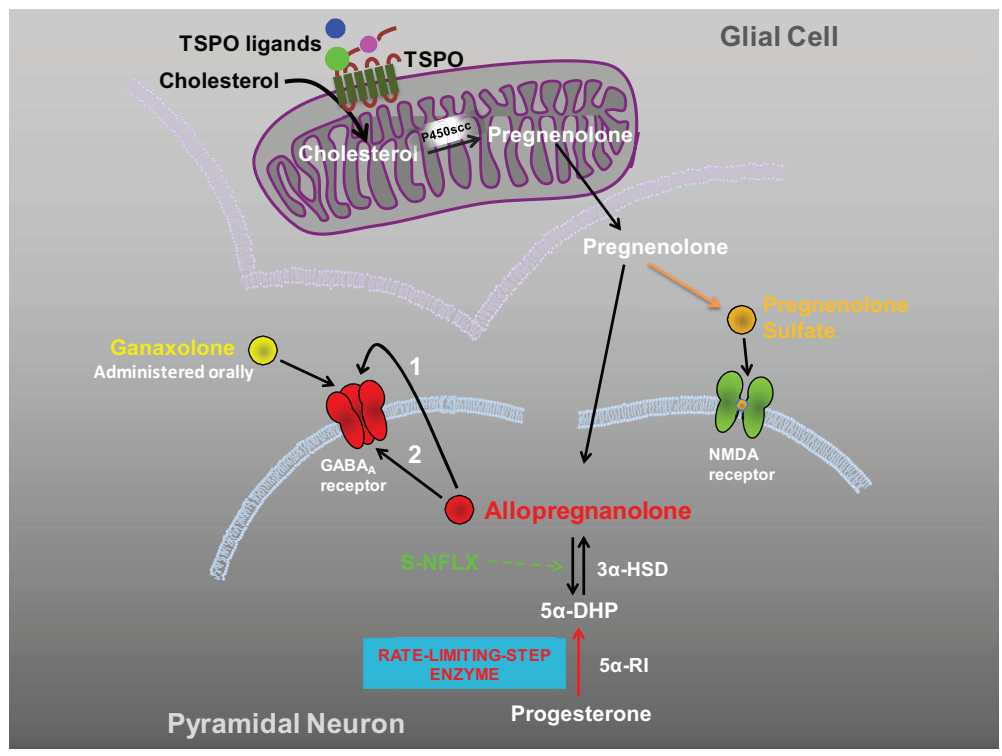

FIGURE 1 | Therapeutic strategies to increase neurosteroidogenesis and improve PTSD by enhancing GABAergic neurotransmission. Depicted are three strategies to improve PTSD symptoms by increasing corticolimbic allopregnanolone levels or by direct activation of $\mathrm{GABA}_{A}$ receptors. (A) TSPO ligands induce an upstream regulation of neurosteroidogenesis by gating the entry of cholesterol into the inner mitochondrial membranes of glial cells, and its conversion into pregnenolone. Pregnenolone can then be taken up by pyramidal neurons (Costa and Guidotti, 1991) where a cascade of enzymatic processes takes place in the cytosol resulting in the production of allopregnanolone. Interestingly, pregnenolone can be further sulfated to pregnenolone sulfate, which has been described as both a positive NMDA receptor modulator (Kussius et al., 2009) and negative $\mathrm{GABA}_{A}$ receptor modulator (Mtchedlishvili and Kapur, 2003). (B) S-NFLX induces a downstream activation of neurosteroidogenesis likely by stimulating allopregnanolone content at the level of $3 \alpha-H S D$ (Griffin and Mellon, 1999). Neurosteroidogenesis is not globally expressed in the brain but relies on rate-limiting step enzymes, which guard allopregnanolone availability and thereby normalize its physiological levels in the required corticolimbic areas (e.g., after activation of TSPO or after S-NFLX). Allopregnanolone, synthesized in glutamatergic cortical or hippocampal pyramidal neurons, may improve PTSD symptoms after being secreted by an autocrine fashion and act locally by binding post-synaptic or extra-synaptic $\mathrm{GABA}_{A}$ receptors located on the same neuron in which it was produced (arrow 1) (Agis-Balboa et al., 2006, 2007). Allopregnanolone may also diffuse into synaptosome membranes of the cell bodies or dendritic arborization to attain intracellular access to specific neurosteroid binding sites of $\mathrm{GABA}_{A}$ receptors (arrow 2) (Akk et al., 2005). (C) Allopregnanolone's analogs (e.g., ganaxolone) directly activate GABA $A_{A}$ receptors and are beneficial in pathological conditions in which allopregnanolone biosynthesis is severely impaired. TSPO, translocate protein (18 kDa); $5 \alpha$-DHP, $5 \alpha$-dihydroprogesterone; $5 \alpha$-RI, $5 \alpha$-reductase type I; $3 \alpha$-HSD, $3 \alpha$-hydroxysteroid dehydrogenase; S-NFLX, S-norfluoxetine.

in which key enzyme blockers for neurosteroid biosynthesis, including finansteride and trilostane (Schüle et al., 2011), were used. TSPO ligands (AC-5216/XBD173 and YL-IPA08) also improve PTSD-like behavior in rodents in studies of situational reminders and contextual fear responses (Qiu et al., 2013). In summary, these studies demonstrated the neuropharmacological effects of several TSPO agents, suggesting that TSPO may represent a therapeutic target for drug discovery. Thus, these drugs, which fulfill the requirements as SBSS molecules, may be a new class of drugs for the future treatment of PTSD and other anxiety disorders. Consistently, TSPO ligands have recently showed promising therapeutic effects in clinical studies (Rupprecht et al., 2010; Schüle et al., 2011).

The advantage of having a drug that "indirectly" activates $\mathrm{GABA}_{\mathrm{A}}$ receptors by increasing allopregnanolone levels Figure 1 within the brain is that allopregnanolone will not be globally increased. Physiological concentrations of allopregnanolone are unevenly expressed in the brain (Pinna et al., 2000; Pibiri et al., 2008), and regulated by rate-limiting step enzymes such as $5 \alpha$-reductase type I.
Pharmacological treatments also induce a cell specific upregulation of brain allopregnanolone, which is increased in frontal cortex (pyramidal neurons, $5 \alpha$-reductase is not expressed in interneurons), hippocampus (CA1-3 pyramidal neurons and dentate gyrus granular cells), and basolateral amygdala (pyramidallike neurons) after fluoxetine but not in striatum (where allopregnanolone is produced in GABAergic long-projecting neurons, spiny neurons) (Agis-Balboa et al., 2006, 2007). Hence, while allopregnanolone is downregulated during social isolation, fluoxetine elevates its levels in glutamatergic neurons but not in GABAergic neurons (Nelson and Pinna, 2011). If allopregnanolone is administered directly, it would be expressed all over the brain and reach high levels in brain regions where its levels are physiologically lower.

Ideally, the SBSS drugs of the future that selectively induce anxiolytic and antiPTSD effects, will be those molecules, prototypic of fluoxetine, devoid of serotonergic effects but capable of activating a neurosteroidogenesis cascade downstream, possibly stimulating allopregnanolone content at the level of $5 \alpha$-reductase or $3 \alpha$-hydroxysteroid dehydrogenase. Understanding whether FLX's action on neurosteroidogenesis is mediated by upregulating expression or function of $5 \alpha$ reductase is of pivotal importance because this enzyme is downregulated in corticolimbic areas of SI mice and in post-mortem frontal cortex (BA9) of depressed patients (Agis-Balboa et al., submitted).

As an alternative, in patients who cannot adequately synthesize allopregnanolone and in whom administration of an SBSS is ineffective because neurosteroidogenesis is greatly impaired, the administration of an allopregnanolone analog (Gulinello et al., 2003; Kaminski et al., 2004), such as ganaxolone that directly activates $\mathrm{GABA}_{\mathrm{A}}$ receptors Figure 1 may offer a safe therapeutic alternative. A multisite Phase II trial of the efficacy and safety of ganaxolone in PTSD is currently under process.

\section{CONCLUSION}

Targeting allopregnanolone biosynthesis with selective neurosteroidogenic agents offers several therapeutic advantages: (1) 
allopregnanolone is not globally expressed in the brain like in the case of administering allopregnanolone itself, in fact, using a neurosteroidogenic molecule relies on the stimulation of rate-limiting step enzymes Figure 1, which guard allopregnanolone levels and thereby normalize its physiological levels in the required brain areas; and (2) stimulating allopregnanolone biosynthesis downstream of pregnenolone in the neurosteroidogenic cascade circumvents the production of several neurosteroids, which by activating various neurotransmitter systems may be associated with unwanted side effects.

\section{ACKNOWLEDGMENTS}

\section{Supported by MH 085999 to Graziano Pinna.}

\section{REFERENCES}

Agis-Balboa, R. C., Pinna, G., Kadriu, B., Costa, E., and Guidotti, A. (2007). Downregulation of $5 \alpha-$ reductase type I mRNA expression in corticolimbic glutamatergic circuits of mice socially isolated for four weeks. Proc. Natl. Acad. Sci. U.S.A. 104, 18736-18741. doi: 10.1073/pnas.0709419104 Agis-Balboa, R. C., Pinna, G., Zhubi, A., Maloku, E., Veldic, M., Costa, E., et al. (2006). Characterization of brain neurons that express enzymes mediating neurosteroid biosynthesis. Proc. Natl. Acad. Sci. U.S.A. 103, 14602-14607. doi: 10.1073/pnas.0606544103

Akk, G., Shu, H. J., Wang, C., Steinbach, J. H., Zorumski, C. F., Covey, D. F., et al. (2005). Neurosteroid access to the $\mathrm{GABA}_{\mathrm{A}}$ receptor. J. Neurosci. 25, 11605-11613. doi: 10.1523/JNEUROSCI.4173-05.2005

Ameli, R., Ip, C., and Grillon, C. (2001). Contextual fear-potentiated startle conditioning in humans: replication and extension. Psychophysiol 38, 383-390. doi: 10.1111/1469-8986.3830383

Belelli, D., and Lambert, J. J. (2005). Neurosteroids: endogenous regulators of the $\mathrm{GABA}_{A}$ receptor. Nat. Rev. Neurosci. 6, 565-575. doi: $10.1038 / \mathrm{nrn} 1703$

Berton, O., and Nestler, I. J. (2006). New approaches to antidepressant drug discovery: beyond monoamines. Nat. Rev. Neurosci. 7, 137-151. doi: $10.1038 / \mathrm{nrn} 1846$

Bremner, J. D., Innis, R. B., Southwick, S. M., Staib, L., Zoghbi, S., and Charney, D. S. (2000). Decreased benzodiazepine receptor binding in prefrontal cortex in combat-related posttraumatic stress disorder. Am. J. Psychiatry 157, 1120-1126. doi: 10.1176/appi.ajp.157.7.1120

Brown, N., Kerby, J., Bonnert, T. P., Whiting, P. J., and Wafford, K. A. (2002). Pharmacological characterization of a novel cell line expressing human alpha(4)beta(3)delta GABA(A) receptors. Br. J. Pharmacol. 136, 965-974. doi: 10.1038/sj.bjp. 0704795

Costa, E., Auta, J., Guidotti, A., Korneyev A., and Romeo, E. (1994). The pharmacology of neurosteroidogenesis. J. Steroid Biochem. Mol. Biol. 49, 385-389. doi: 10.1016/0960-0760(94)90284-4

Costa, E., and Guidotti, A. (1991). Diazepam binding inhibitor (DBI): a peptide with multiple biological actions. Life Sci.49, 325-344. doi: 10.1016/00243205(91)90440-M

Forbes, D., Parslow, R., Creamer, M., Allen, N., McHugh, T., and Hopwood, M. (2008). Mechanisms of anger and treatment outcome in combat veterans with posttraumatic stress disorder. J. Trauma. Stress 21, 142-149. doi: $10.1002 /$ jts. 20315

Griffin, L. D., and Mellon, S. H. (1999). Selective serotonin reuptake inhibitors directly alter activity of neurosteroidogenic enzymes. Proc. Natl. Acad. Sci. U.S.A. 96, 13512-13517. doi: 10.1073/pnas.96.23.13512

Guidotti, A., and Costa, E. (1998). Can the antidysphoric and anxiolytic profiles of selective serotonin reuptake inhibitors be related to their ability to increase brain 3 alpha, 5alphatetrahydroprogesterone (allopregnanolone) availability? Biol. Psychiatry 44, 865-873. doi: 10.1016/S0006-3223(98)00070-5

Gulinello, M., Gong, Q. H., and Smith, S. S. (2003). Progesterone withdrawal increases the anxiolytic actions of gaboxadol: role of alpha4betadelta GABA(A) receptors. Neuroreport 14, 43-46. doi: 10.1097/00001756-200301200-00008

Ipser, J. C., and Stein, D. J. (2012). Evidence-based pharmacotherapy of post-traumatic stress disorder (PTSD). Int. J. Neuropsychopharmacol. 15, 825-840. doi: 10.1017/S1461145711001209

Joseph, J. S., and Gray, M. J., (2008). Exposure therapy for posttraumatic stress disorder. J. Behav. Anal. Offender Vict. 1, 69-80.

Kaminski, R. M., Livingood, M. R., and Rogawski, M. A. (2004). Allopregnanolone analogs that positively modulate GABA receptors protect against partial seizures induced by $6-\mathrm{Hz}$ electrical stimulation in mice. Epilepsia 45, 864-867. doi: 10.1111/j.0013-9580.2004.04504.x

Kita, A., Kohayakawa, H., Kinoshita, T., Ochi, Y., Nakamichi, K., Kurumiya, S., et al. (2004). Antianxiety and antidepressant-like effects of AC-5216, a novel mitochondrial benzodiazepine receptor ligand. Br. J. Pharmacol. 142, 1059-1072. doi: 10.1038/sj.bjp.0705681

Kugaya, A., Sanacora, G., Verhoeff, N. P., Fujita, M., Mason, G. F., Seneca, N. M., et al. (2003). Cerebral benzodiazepine receptors in depressed patients measured with [123I]iomazenil SPECT. Biol. Psychiatry 54, 792-799. doi: 10.1016/S00063223(02)01788-2

Kussius, C. L., Kaur, N., and Popescu, G. K. (2009). Pregnanolone sulfate promotes desensitization of activated NMDA receptors. J. Neurosci. 29, 6819-6827. doi: 10.1523/JNEUROSCI.028109.2009

Lovick, T. (2013). SSRIs and the female brain potential for utilizing steroid-stimulating properties to treat menstrual cycle-linked dysphorias. J. Psychopharmacol. 27, 1180-1185. doi: $10.1177 / 0269881113490327$

Maren, S. (2008). Pavlonian fear conditioning as a behavioral assay for hippocampus and amygdala function: cautions and caveats. Eur. J. Neurosci. 28, 1661-1666. doi: 10.1111/j.14609568.2008.06485.x
Marks, I. (1979). Exposure therapy for phobias and obsessive-compulsive disorders. Hosp. Pract. 14, 101-108.

Mihalek, R. M., Banerjee, P. K., Korpi, E. R., Quinlan, J. J., Firestone, L. L., Mi, Z. P., et al. (1999). Attenuated sensitivity to neuroactive steroids in gamma-aminobutyrate type A receptor delta subunit knockout mice. Proc. Natl. Acad. Sci. U.S.A. 96, 12905-12910. doi: 10.1073/pnas.96.22. 12905

Mtchedlishvili, Z., and Kapur, J. (2003). A presynaptic action of the neurosteroid pregnenolone sulfate on GABAergic synaptic transmission. Mol. Pharmacol. 64, 857-864. doi: 10.1124/mol.64.4.857

Myers, K. M., and Davis, M. (2007). Mechanisms of fear extinction. Mol. Psychiatry 12, 120-150. doi: 10.1038/sj.mp.4001939

Nelson, M., and Pinna, G. (2011). S-norfluoxetine microinfused into the basolateral amygdala increases allopregnanolone levels and reduces aggression in socially isolated mice. Neuropharmacology 27, 1180-1185. doi: 10.1016/ j.neuropharm.2010.10.011

Nin, S. M., Martinez, L. A., Thomas, R., Nelson, M., and Pinna, G. (2011). Allopregnanolone and Snorfluoxetine decrease anxiety-like behavior in a mouse model of anxiety/depression. Trabajos del Instituto Cajal 83, 215-216. doi: 10.3389/fendo. 2011.00073

Papadopoulos, V., Baraldi, M., Guilarte, T. R., Knudsen, T. B., Lacapère, J. J., Lindemann, P., et al. (2006). Translocator protein $(18 \mathrm{kDa})$ : new nomenclature for the peripheral-type benzodiazepine receptor based on its structure and molecular function. Trends Pharmacol. Sci. 27, 402-409. doi: 10.1016/j.tips.2006.06.005

Pibiri, F., Nelson, M., Guidotti, A., Costa, E., and Pinna, G. (2008). Decreased allopregnanolone content during social isolation enhances contextual fear: a model relevant for posttraumatic stress disorder. Proc. Natl. Acad. Sci. U.S.A. 105, 5567-5572. doi: 10.1073/pnas.0801853105

Pinna, G. (2010). In a mouse model relevant for post-traumatic stress disorder, selective brain steroidogenic stimulants (SBSS) improve behavioral deficits by normalizing allopregnanolone biosynthesis. Behav. Pharmacol. 21, 438-450. doi: 10.1097/FBP.0b013e $32833 \mathrm{~d} 8 \mathrm{ba} 0$

Pinna, G., Agis-Balboa, R., Pibiri, F., Nelson, M., Guidotti, A., and Costa, E. (2008). Neurosteroid biosynthesis regulates sexually dimorphic fear and aggressive behavior in mice. Neurochem. Res. 33, 1990-2007. doi: 10.1007/s11064008-9718-5

Pinna, G., Costa, E., and Guidotti, A. (2004). Fluoxetine and norfluoxetine stereospecifically facilitate pentobarbital sedation by increasing neurosteroids. Proc. Natl. Acad. Sci. U.S.A. 101, 6222-6225. doi: 10.1073/pnas.0401479101

Pinna, G., Costa, E., and Guidotti, A. (2006a). Fluoxetine and norfluoxetine stereospecifically and selectively increase brain neurosteroid content at doses that are inactive on 5-HT reuptake. Psychopharmacology 186, 362-372. doi: 10.1007/s00213-005-0213-2

Pinna, G., Agis-Balboa, R. C., Zhubi, A., Matsumoto, K., Grayson, D. R., Costa, E., et al. (2006b) 
Imidazenil and diazepam increase locomotor activity in mice exposed to protracted social isolation. Proc. Natl. Acad. Sci. U.S.A. 103, 4275-4280. doi: 10.1073/pnas.0600329103

Pinna, G., Costa, E., and Guidotti, A. (2009). SSRIs act as selective brain steroidogenic stimulants (SBSSs) at low doses that are inactive on 5HT reuptake. Cur. Opin. Pharmac. 9, 24-30. doi: 10.1016/j.coph.2008.12.006

Pinna, G., Dong, E., Matsumoto, K., and Costa, E, and Guidotti, A. (2003). In socially isolated mice, the reversal of brain allopregnanolone downregulation mediates the anti-aggressive action of fluoxetine. Proc. Natl. Acad. Sci. U.S.A. 100, 2035-2040. doi: 10.1073/pnas.0337642100

Pinna, G., and Rasmusson, A. M. (2012). Upregulation of neurosteroid biosynthesis as a pharmacological strategy to improve behavioural deficits in a putative mouse model of posttraumatic stress disorder. J. Neuroendocrinol. 24, 102-116. doi: 10.1111/j.1365-2826.2011. 02234.x

Pinna, G., Uzunova, V., Matsumoto, K., Puia, G., Costa, E., and Guidotti, A. (2000). Brain allopregnanolone regulates the potency of the $\mathrm{GABA}_{A}$ receptor agonist muscimol. Neuropharmacology 39, 440-448. doi: 10.1016/S0028-3908(99)00149-5

Qiu, Z. K., Zhang, L. M., Zhao, N., Chen, H. X., Zhang, Y. Z., Liu, Y. Q., et al. (2013). Repeated administration of AC-5216, a ligand for the $18 \mathrm{kDa}$ translocator protein, improves behavioral deficits in a mouse model of post-traumatic stress disorder. Prog. Neuropsychopharmacol. Biol. Psychiatry 45, 40-46. doi: 10.1016/j.pnpbp.2013.04.010

Rasmusson, A. M., Pinna, G., Paliwal, P., Weisman, D., Gottshalk, C., Charney, D., et al. (2006). Decreased cerebrospinal fluid allopregnanolone levels in women with posttraumatic stress disorder. Biol. Psychiatry 60, 704-713. doi: 10.1016/j.biopsych.2006.03.026

Rauch, S. L., Shin, L. M., and Phelps, E. A. (2006) Neurocircuitry models of posttraumatic stress disorder and extinction: human neuroimaging research-past, present, and future. Biol. Psychiatry 60, 376-382. doi: 10.1016/j.biopsych.2006.06.004

Romeo, E., Ströhle, A., Spalletta, G., di Michele, F., Hermann, B., Holsboer, F., et al. (1998). Effects of antidepressant treatment on neuroactive steroids in major depression. Am. J. Psychiatry 155 , 910-913.

Rupprecht, R., Papadopoulos, V., Rammes, G., Baghai, T. C., Fan, J., Akula, N., et al. (2010). Translocator protein $(18 \mathrm{kDa})$ (TSPO) as a therapeutic target for neurological and psychiatric disorders. Nat. Rev. Drug Discov. 9, 971-988. doi: 10.1038/nrd3295

Rupprecht, R., Rammes, G., Eser, D., Baghai, T. C., Schüle, C., Nothdurfter, C., et al. (2009). Translocator protein (18 kD) as target for anxiolytics without benzodiazepinelike side effects. Science 325, 490-493. doi: 10.1126/science. 1175055

Schüle, C., Eser, D., Baghai, T. C., Nothdurfter, C., Kessler, J. S., and Rupprecht, R. (2011). Neuroactive steroids in affective disorders: target for novel antidepressant or anxiolytic drugs? Neuroscience 191, 55-77. doi: 10.1016/j.neuroscience.2011.03.025

Steiner, M., and Pearlstein, T. (2000). Premenstrual dysphoria and the serotonin system: pathophysiology and treatment. J. Clin. Psychiatry 12, 17-21.

Stell, B. M., Brickley, S. G., Tang, C. Y., Farrant, M., and Mody, I. (2003). Neuroactive steroids reduce neuronal excitability by selectively enhancing tonic inhibition mediated by delta subunit-containing GABA $_{A}$ receptors. Proc. Natl. Acad. Sci.
U.S.A. 100, 14439-14444. doi: 10.1073/pnas. 2435457100

Uzunov, D. P., Cooper, T. B., Costa, E., and Guidotti, A. (1996). Fluoxetine elicited changes in brain neurosteroid content measured by negative ion mass fragmentography. Proc. Natl. Acad. Sci. U.S.A. 93, 12599-12604. doi: 10.1073/pnas.93.22. 12599

Uzunova, V., Sheline, Y., Davis, J. M., Rasmusson, A., Uzunov, D. P., Costa, E., et al. (1998). Increase in the cerebrospinal fluid content of neurosteroids in patients with unipolar major depression who are receiving fluoxetine or fluvoxamine. Proc. Natl. Acad. Sci. U.S.A. 95, 3239-3244. doi: 10.1073/pnas.95.6.3239

Westenberg, H. G. M. (1996). Development in the drug treatment of panic disorder: what is the place of the selective serotonin reuptake inhibitors? J. Affec. Dis. 40, 85-93. doi: 10.1016/01650327(96)00043-2

Received: 17 October 2013; accepted: 14 December 2013; published online: 06 January 2014

Citation: Pinna G (2014) Targeting neurosteroidogenesis as therapy for PTSD. Front. Pharmacol. 4:166. doi: 10.3389/fphar.2013.00166

This article was submitted to Neuropharmacology, a section of the journal Frontiers in Pharmacology. Copyright (c) 2014 Pinna. This is an open-access article distributed under the terms of the Creative Commons Attribution License (CC BY). The use, distribution or reproduction in other forums is permitted, provided the original author(s) or licensor are credited and that the original publication in this journal is cited, in accordance with accepted academic practice. No use, distribution or reproduction is permitted which does not comply with these terms. 